\title{
Integer Points close to Convex Hypersurfaces
}

\author{
M. C. Lettington* \\ Preprint, 2010
}

\section{Introduction}

Let $C$ be the boundary surface of a strictly convex bounded $d$-dimensional body. Strictly convex means that if $P$ and $Q$ are points on $C$, then points on the line segment $P Q$ between $P$ and $Q$ lie in the convex body, but not on its boundary $C$. Let $M C$ denote the dilation of $C$ by a factor $M$. Andrews [1], [2], proved that the number of points of the integer lattice on $M C$ is

$$
O\left(M^{\frac{d(d-1)}{d+1}}\right)
$$

as $\mathrm{M}$ tends to infinity. Strict convexity is necessary because a part of a $(d-1)$ dimensional hyperplane in the boundary $C$ can give as many as a constant times $M^{d-1}$ integer points for infinitely many values of $M$.

We consider the integer points within a distance $\delta$ of the hypersurface $M C$. The two-dimensional case has been well studied [12], [5], [9], [6], [10] and [11]. More recently the author has examined the three-dimensional case [15]. Introducing $\delta$ requires some uniform approximability condition on the surface $C$, usually expressed in terms of upper and lower bounds for derivatives and determinants of derivatives. Let $A$ be the $(d-1)$-dimensional volume of $C$. The search region has $d$-dimensional volume

$$
\left(2 A \delta+O\left(\delta^{2}\right)\right) M^{d-1}
$$

and this is known to be the number of integer points on average over translations of the surface $M C$. To obtain an asymptotic formula one considers the Fourier transform of the convex body, with conditions at least as far as the $6 d$-th derivatives in order to estimate the multiple exponential integrals. Hlawka [8] obtained an asymptotic formula with error of size (1); see also Krätzel [13]. Under the

2000 Mathematics Subject Classification: Primary 11P21; Secondary 11H06, 11J25.

* This paper forms part of my PhD thesis in the University of Wales.

I would like to thank my supervisor Professor M N Huxley for all his invaluable support and guidance in this problem. 
$C^{\infty}$ hypothesis of a convergent Taylor series, the error term in the asymptotic formula has been improved, most recently by Müller [18].

We derive an upper bound for the number of integer points within a distance $\delta$ of the hypersurface. We require only that $C$ has a tangent hyperplane at every point, and that any two-dimensional cross-section through the normal at some point $P$ consists (in a neighbourhood of $P$ ) of a plane curve $C^{\prime}$ with continuous radius of curvature bounded away from zero and infinity.

CURVATURE CONDITION (with size parameter $M$ ). For any point $P$ on $C$ and any two-plane $\Pi$ through the normal to $C$ at $P$, let $C(\Pi, P)$ be the closed plane curve $C \cap \Pi$. Then $C(\Pi, P)$ is a twice differentiable plane curve with radius of curvature $\rho$ lying in the range

$$
c_{0} M+\frac{1}{2} \leq \rho \leq c_{1} M-\frac{1}{2}
$$

where the constants $c_{0}, c_{1}$ and $\delta$ satisfy

$$
\frac{1}{M}<c_{0} \leq 1 \leq c_{1} \text {, and } \delta<\frac{1}{4}
$$

LOCAL CURVATURE CONDITION. There is a constant $\kappa$ such that for $C(\Pi, P)$ defined as above, the points $Q$ of $C(\Pi, P)$ with $P Q \leq \kappa M$ form a twice differentiable plane curve with radius of curvature satisfying (3).

In order to state our results, we set up some notation. Let $C_{0}$ be the locus of points at distance $\delta$ from $C$ measured along the interior normals to $C$, and let $C_{1}$ be the locus of points at distance $\delta$ measured along the exterior normals. Let $E$ be the $d$-dimensional shell bounded by $C_{0}$ and $C_{1}$ so that $E$ has thickness $2 \delta$. Let $S$ be the set of integer points in $E$, and let $H$ be the convex hull of $S$, so that $H$ is a $d$-dimensional convex polytope [3], [4], [14], [16] and [17]. All points of $S$ lie in $H$, but not all integer points on the boundary of $H$ lie in $S$.

By Lemma 2.1 of [15], the boundary surfaces $C_{0}$ and $C_{1}$ of the shell $E$ have a tangent hyperplane at each point $Q$, and their two-dimensional cross-sections $C(\Pi, Q)$ in planes normal to the tangent hyperplanes are twice differentiable, with radius of curvatures in the range

$$
c_{0} M \leq \rho \leq c_{1} M
$$

Under the Curvature Condition, the shell $E$ containing $S$, the set of integer points, lies in a $d$-hypersphere of radius $R=c_{1} M$. The volume $V_{d}$ and surface content $S_{d}$ of this sphere is given by the formulae [19]

$$
V_{d}=\alpha_{d} R^{d}, \quad S_{d}=d \alpha_{d} R^{d-1}
$$


where

$$
\alpha_{2 k}=\frac{\pi^{k}}{k !}, \quad \alpha_{2 k+1}=\frac{2^{2 k+1} \pi^{k} k !}{(2 k+1) !}, \quad \alpha_{d} \leq 6, \quad \frac{\alpha_{d}}{\alpha_{d-1}} \leq \pi,
$$

and for $d \geq 2$,

$$
d \alpha_{d} \leq(2 \pi)^{d-1}
$$

We can now state our results.

THEOREM 1.1. Suppose that $C$ is a convex hypersurface in d-dimensional Euclidean space $\mathbb{E}^{d}(d \geq 3)$, satisfying the Curvature Condition at size $M$ (so that $C$ is contained in a hypersphere radius $\left.c_{1} M\right)$. Then the total number, $N$, of integer points lying either on $C$, or within a distance $\delta$ of $C$, is bounded by

$$
N \leq \frac{2^{3 d^{2}+5 d-7} d !}{\alpha_{d-1}}\left(\frac{c_{1}}{c_{0}}\right)^{d-1}\left(\left(c_{1} M\right)^{\frac{d(d-1)}{d+1}}+2^{9} \delta\left(c_{1} M\right)^{d-1}\right) .
$$

THEOREM 1.2. Suppose that $C$ is a convex hypersurface in d-dimensional Euclidean space $\mathbb{E}^{d}(d \geq 3)$, satisfying the Local Curvature Condition at size $M$ (so that $C$ is contained in a hypersphere radius $c_{1} M$ ), with

$$
M \geq \frac{100 \delta c_{1}}{\kappa^{2}}
$$

Then $N$, the total number of integer points lying either on $C$, or within a distance $\delta$ of $C$, satisfies the same bound (9) as in Theorem 1.1.

\section{Major Arcs}

DEFINITION (major and minor arcs). It is helpful in many problems to separate "major arcs", regions where there is good Diophantine approximation, from "minor arcs", regions where there is not. In this paper a major arc can be described informally as a region $U$ of the shell $E$ such that the convex hull of all the integer points in $U$ is contained in the intersection of $E$ with some hyperplane. Hence $U$ can be of dimension $j$, with $j=1,2, \ldots, d-1$.

For each major arc we are interested in the integer points which lie within a distance $\delta$ from the hypersurface $C$. In the preceding paper [15] we showed that the integer points lie in clusters around the vertices of the convex hull $H$, which we call components of a major arc. We also observed that at most two onedimensional components can lie on the same straight line. Higher dimensional components, are however, not as simple and for $d-1 \geq j \geq 2$, there can exist many $j$-dimensional components on the same $j$-dimensional plane.

Each $j$-dimensional component of a major arc has maximum diameter equal to the maximum length of a component of a one-dimensional major arc. By Lemma 4.1 of [15] this is

$$
\leq 4 \sqrt{\delta c_{1} M}
$$


Hence a $j$-dimensional component is contained within a $j$-dimensional hypercube of volume

$$
\leq\left(4 \sqrt{\delta c_{1} M}\right)^{j}
$$

LEMMA 2.1. Let $\Pi$ be a hyperplane with equation

$$
\mathbf{n} \cdot \mathbf{x}=D,
$$

where $\mathbf{n}$ is a primitive integer vector, and $D$ is an integer. Then the integer points of $\Pi$ form a lattice with determinant $|\mathbf{n}|$.

Proof. This is Lemma 4.4 of [15].

LEMMA 2.2. Let $\Lambda$ be a $j$-dimensional lattice of determinant $n, 1 \leq j \leq d$. Let $U$ be a convex set in the $j$-plane of $\Lambda$, with $j$-dimensional volume $V$, containing $K$ points of the lattice $\Lambda$. Then one of the following two cases holds.

(1) Major case. All the points of $\Lambda$ in the set $U$ lie on a $(j-1)$-dimensional plane.

(2) Minor case.

$$
K \leq j ! \frac{V}{n}+j \leq(j+1) ! \frac{V}{n} .
$$

Proof. This is Lemma 4.5 of [15].

\section{Vertex Components}

For each point $P$ in our shell $E$, there exists a normal to the hypersurface $C$, meeting the outer boundary $C_{1}$ normally at a point $R_{1}$ and the inner boundary surface $C_{0}$ normally at a point $R_{0}$. We call $R_{0}$ and $R_{1}$ the normal projections of $P$ onto $C_{0}$ and $C_{1}$ respectively. The vertices of our convex polytope $H$, must, by definition lie in $E$ and for every other non-vertex integer point in $E$ there must exist a nearest vertex. This argument follows the account in [15] of the 3-dimensional case.

DEFINITION (vertex components). Let $P$ be a point of $S$ in the shell $E$ and $R_{1}$ the normal projection of $P$ onto $C_{1}$. Let $V$ be a vertex of the convex hull $H$ and $E^{\prime}$ the plane sectional strip of $E$ containing $V, P$ and $R_{1}$. If the line segment $R_{1} V$ lies entirely within the closed strip $E^{\prime}$, then we say that $P$ lies in the component $S(V)$ of $S$.

LEMMA 3.1. Every point $P$ of $S$ belongs to some vertex component $S(V)$. 
Proof. The line segment $P R_{1}$ cuts the boundary of the convex hull $H$ at some point $Q$ between $P$ and $R_{1}$ inside $E$, so that $Q$ lies in some hyperplane face $F$ of $H$. If $Q$ is a vertex of $H$ then $P$ belongs to $S(Q)$ as $Q R_{1}$ will lie on the line segment $R_{0} R_{1}$ inside $E$.

We now assume that the points $Q$ is not a vertex of $H$ and triangulate the facet $F$ of $H$ containing $Q$ so that $Q$ lies in some simplex $W=V_{1} V_{2} V_{3} \ldots V_{d}$. If the line segment $Q V_{i}$ does not enter the interior of the convex set bounded by $C_{0}$ then neither does $R_{1} V_{i}$, implying that $P$ lies in $S\left(V_{i}\right)$.

If $P$ lies in no $S\left(V_{i}\right)$ then each line segment $Q V_{i}$ on $F$ cuts the interior of $C_{0}$ in some point $Q_{i}$ also on $F$ but not in $E$. The whole convex simplex $Q_{1} Q_{2} \ldots Q_{d}$ therefore lies strictly inside $C_{0}$ and contains $Q$. Hence, $Q$ is not in $E$ which is impossible, since $Q$ lies on the line segment $R_{0} R_{1}$, which is strictly inside $E$. This contradiction shows that for some $i$, the line segment $V_{i} Q$ lies in $E$ and so $V_{i} R_{1}$ lies in $E$ and $P$ is in the component corresponding to $V_{i}$.

LEMMA 3.2 (spacing lemma). Let $V$ be a vertex of the convex hull $H$. Let $P$ be a point of $S$ not in the component $S(V)$ of $V$. Let $R_{1}$ and $R_{2}$ be the respective normal projections of $P$ and $V$ onto $C_{1}$. Then

$$
R_{1} R_{2}>\sqrt{c_{0} \delta M}
$$

and the angle between the normals to $C_{1}$ at $R_{1}$ and $R_{2}$ is

$$
>\frac{1}{c_{1}} \sqrt{\frac{c_{0} \delta}{M}}
$$

Proof. This is Lemma 5.2 of [15]. The number of dimensions does not affect the geometry of the 2-dimensional section.

As each integer point $P$ in $S$ belongs to at least one component $S(V)$ labelled by some vertex $V$ of the convex hull $H$, components labelled by different vertices may well overlap and different vertices of the convex hull may be close together. We pick a well-spaced set of vertices of $H$ as follows. Pick a vertex $V_{1}$, and let the enlarged component $S^{\prime}\left(V_{1}\right)$ be the union of all components $S(V)$ with $V$ in $S\left(V_{1}\right)$.

Now pick a vertex $V_{2}$ not in $S^{\prime}\left(V_{1}\right)$, and form the enlarged component $S^{\prime}\left(V_{2}\right)$. We pick $V_{i+1}$ not in $S^{\prime}\left(V_{1}\right), S^{\prime}\left(V_{2}\right), \ldots, S^{\prime}\left(V_{i}\right)$, and so on until all of the vertices $V$ of the convex hull $H$ lie in some enlarged component.

LEMMA 3.3 (thickness lemma). Let $S^{\prime}(V)$ be an enlarged component and let $R_{2}$ be the normal projection of $V$ onto $C_{1}$. Let $P$ be a point in $S^{\prime}(V)$ and let $R_{1}$ be the normal projection of $P$ onto $C_{1}$. Then the distance $h$ of $P$ from the tangent plane at $R_{2}$ satisfies

$$
h \leq \frac{52 \delta c_{1}}{c_{0}} .
$$


and

$$
R_{1} R_{2} \leq 10 \sqrt{\delta c_{1} M}
$$

Proof. This is Lemma 5.3 of [15]. The number of dimensions does not affect the geometry of the 2-dimensional section.

REMARK. As with the three-dimensional case in [15], we are ultimately working towards a shelling argument. This uses the property that if we can obtain a bound valid for $\delta$ sufficiently small, then we can deduce a possible weaker bound for large $\delta$ by dividing the shell $E$ into concentric shells $E_{r}, 1 \leq r \leq R$ of thickness $\delta_{0}$, bounded by shrunken copies of the exterior hypersurface $C_{1}$ of $E$. By inequality (5), we have a uniform upper bound of $c_{1} M$ for the sectional radius of curvature at any point on each shell $E_{r}$. Hence, when regarding maximum sectional radius of curvatures, we can work within the general shell boundary $C_{1}$, whose sectional radius of curvature is also $\leq c_{1} M$.

LEMMA 3.4 (flatness lemma). Let $S^{\prime}(V)$ be an enlarged vertex component of our convex hull $H$. If

$$
\delta<\delta_{0}=\left(\frac{c_{0}}{2^{2 d} 5^{d-1} 13 d ! c_{1}}\right)^{\frac{2}{d+1}}\left(c_{1} M\right)^{\frac{-(d-1)}{d+1}},
$$

then all the points of $S^{\prime}(V)$ lie on a hyperplane through the vertex $V$.

Proof. Let $P$ be a point of $S^{\prime}(V)$ and let $R_{1}$ and $R_{2}$ be the the normal projections of $P$ and $V$ onto $C_{1}$. All points $P$ of $S^{\prime}(V)$ lie within a distance $52 \delta c_{1} / c_{0}$ from the tangent hyperplane at $R_{2}$ and by $(16)$

$$
P V \leq R_{1} R_{2} \leq 10 \sqrt{\delta c_{1} M}
$$

Hence, the set of integer points $S^{\prime}(V)$ all lie within a rectangular box $L$, of $d$ dimensional volume $\operatorname{Vol}(L)$, with

$$
\operatorname{Vol}(L) \leq \frac{52 \delta c_{1}}{c_{0}}\left(20 \sqrt{\delta c_{1} M}\right)^{d-1}<\frac{1}{d !}
$$

where we have used the assumption (17). Therefore, by Lemma 2.2, the major arc case holds, and all points of the enlarged vertex component $S^{\prime}(V)$, including $V$ itself, lie on a hyperplane.

LEMMA 3.5 (approximate tangency). Let $S^{\prime}(V)$ be an enlarged component. Let $T$ be the point of $C_{1}$ closest to $V$. Let $P$ be another point of $S^{\prime}(V)$, and let $\mathbf{g}$ be the integer vector $V P$. Then the angle $\alpha$ between $V P$ and the normal to $C_{1}$ at $T$ satisfies

$$
|\cos \alpha| \leq \frac{52 \delta c_{1}}{c_{0}|\mathbf{g}|}
$$


Proof. This is Lemma 5.5 of [15]. The number of dimensions does not affect the geometry of the 2-dimensional section.

LEMMA 3.6 (sums of reciprocal vector lengths). For $j=1, \cdots, d-1$ we have

$$
\sum_{1 \leq|\mathbf{e}| \leq E} \frac{1}{|\mathbf{e}|^{j}} \leq 2^{2 d+j} E^{d-j} .
$$

Proof. Applying the Cauchy condensation method, we divide the normal vectors into ranges

$$
\frac{F}{2}<|\mathbf{e}| \leq F, \quad F=1,2,4, \ldots, 2^{K},
$$

where $2^{K}$ is the largest power of 2 less than or equal to $E$. The number of integer vectors in this range is

$$
\begin{gathered}
\leq(2 F+1)^{d}-(F+1)^{d} \leq \sum_{r=0}^{d}\left(\begin{array}{l}
d \\
j
\end{array}\right)\left(2^{d-j}-1\right) F^{d-j} \\
\leq F^{d}\left(3^{d}-2^{d}\right) \leq 2^{2 d-1} F^{d},
\end{gathered}
$$

so that

$$
\sum_{F / 2<|\mathbf{e}| \leq F} \frac{1}{|\mathbf{e}|^{j}} \leq 2^{2 d-1} F^{d} \cdot\left(\frac{2}{F}\right)^{j}=2^{2 d+j-1} F^{d-j} .
$$

Summing over the ranges for $\mathrm{F}$, we have

$$
\begin{gathered}
\sum_{1 \leq|\mathbf{e}| \leq F} \frac{1}{|\mathbf{e}|^{j}} \leq 2^{2 d+j-1}\left(1+\left(2^{1}\right)^{d-j}+\left(2^{2}\right)^{d-j}+\ldots+\left(2^{K}\right)^{d-j}\right) \\
=2^{2 d+j-1} \frac{\left(2^{d-j}\right)^{k+1}-1}{2^{d-j}-1} \leq 2^{2 d+j} 2^{(d-j) K} \\
\leq 2^{2 d+j} E^{d-j}
\end{gathered}
$$

DEFINITION (the reach of an Enlarged Vertex Component). Let $R$ be the normal projection of $V$ onto the outer surface $C_{1}$. We define the reach, $U(V)$, of the enlarged vertex component $S^{\prime}(V)$ to be the set of points on $C_{1}$ such that for all points $P \in U(V)$ we have

$$
P R \leq 10 \sqrt{\delta c_{1} M}
$$

By (16), if $Q$ is an integer point in $S^{\prime}(V)$, the normal projection $R_{1}$ of $Q$ onto the surface $C_{1}$ lies in $U(V)$, the reach of the enlarged component $S^{\prime}(V)$. 
LEMMA 3.7 (Enlarged Vertex Components and the Local Curvature Condition). If

$$
M \geq \frac{100 \delta c_{1}}{\kappa^{2}}
$$

then the Local Curvature Condition with respect to $R$, holds at all points $R_{1}$ in the reach of $S^{\prime}(V)$.

Proof. Let $P$ be a point of $C_{1}$ in $U(V)$. By (21) and (22)

$$
P R \leq 10 \sqrt{\delta c_{1} M} \leq \kappa M
$$

which is the threshhold for the Local Curvature Condition.

LEMMA 3.8. In d-dimensional space, the number of integer points of $S$ in $E$ that lie strictly inside the convex hull $H$ of $S$ is

$$
\leq 2 \delta d ! \alpha_{d} d\left(c_{1} M\right)^{d-1}
$$

Proof. This is Lemma 4.3 of [15].

Let $S(H)$ be the set of integer points in $S$ that lie on the boundary of the convex hull $H$. The rest of this paper is devoted to the study of $S(H)$. The points of $S(H)$ fall into enlarged vertex components, where an enlarged vertex component, $S^{\prime}(V)$ of $S(H)$, is either full $d$-dimensional or it lies strictly on some $j$-dimensional hyperplane that contains the vertex $V$, with $0 \leq j \leq d-1$.

LEMMA 3.9. Let $f_{d-1}$ be the number of $(d-1)$-dimensional hyperplane faces of the convex hull $H$. Then

$$
f_{d-1} \leq 2\left(3 \alpha_{d} d !\right)^{\frac{d}{d+1}}\left(c_{1} M\right)^{\frac{d(d-1)}{d+1}} \leq 36 d !\left(c_{1} M\right)^{\frac{d(d-1)}{d+1}} .
$$

Proof. This is Theorem 3.4 of [15], where we have used (7) to obtain the second inequality.

LEMMA 3.10. For $0 \leq j \leq(d-2)$, let $f_{j}$ be the number of $j$-faces $(j$ dimensional faces) of the convex hull $H$. Then

$$
f_{j} \leq 2\left(3 \alpha_{d} d !\right)^{\frac{d}{d+1}}\left(2(j+1) c_{1} M\right)^{\frac{d(d-1)}{d+1}} \leq 36 d !\left(2(j+1) c_{1} M\right)^{\frac{d(d-1)}{d+1}} .
$$

Proof. This is Theorem 3.6 of [15].

LEMMA 3.11. Let $R=c_{1} M$ and let $F$ be a facet or hyperplane face of $H$ that lies in a hyperplane $\Psi$ with outward normal $\mathbf{n}$. Let $X$ be the point of $C_{1}$ at which $\mathbf{n}$ is the outward normal. Let $h$ be the distance from $X$ along the inward normal to the nearest point $Y$ on the hyperplane $\Psi$. Let $E^{\prime}$ be the $(d-1)$-dimensional section of $E$ contained in $\Psi$, so that $E^{\prime}$ contains all parts of the face $F$ that lie in the shell $E$. Then the $(d-1)$-dimensional volume $V$ of $E^{\prime}$ is bounded above by

$$
V \leq 2^{\frac{d+9}{2}} d \delta\left(c_{1} M\right)^{\frac{d-1}{2}} h^{\frac{d-3}{2}} .
$$

Proof. This is Lemma 4.2 of [15]. 


\section{Boundary Components}

Let $S^{\star}\left(V_{i}\right)$ be the subset of $S^{\prime}\left(V_{i}\right)$ consisting of integer points on the boundary of $H$. We will call this a boundary component. We have shown that for each enlarged vertex component $S^{\prime}\left(V_{i}\right)$, if $\delta$ is sufficiently small then $S^{\prime}\left(V_{i}\right)$ lies in a hyperplane and so $S^{\star}\left(V_{i}\right)$ lies in the same hyperplane.

The dimension of the integer point set $S^{\star}\left(V_{i}\right)$ is defined to be the least $e$ for which $S^{\star}\left(V_{i}\right)$ lies in an $e$-dimensional hyperplane and $\left|S^{\star}\left(V_{i}\right)\right|$ to be the number of elements of $S^{\star}\left(V_{i}\right)$ in $S$. When $e=0$ we merely have to count the vertices of $H$. When $e=d$, the points of the enlarged vertex component lie on two or more hyperfaces of $H$, and we use a volume argument (Lemma 4.3 below). When $e=d-1$ we have a straightforward but complicated estimation (Lemma 4.2 below). For intermediate dimensions $1 \leq e \leq d-2$ we consider "girdles" of parallel planes and use a solid angle spacing argument. This takes its simplest form when $e=1$ (Lemma 4.1 below). The cases $2 \leq e \leq d-2$ require more combinatorial geometry and will be considered in the next section.

We define a one-dimensional girdle to be the set of all the boundary components $S^{\star}(V)$ of $H$ which are one-dimensional and which lie parallel to some primitive integer vector e. When considering the $j$-dimensional boundary components with $j \leq d-2$, we must also take into account the possibility that many of these components may be parallel. To clarify the parallel condition in higher dimensions, we introduce the idea of degrees of parallelism as described in [19].

DEFINITION (degrees of parallelism in higher dimensions). Let $\Pi_{1}$ and $\Pi_{2}$ be two planes of dimension $p$ and $q(p \geq q)$ respectively in $\mathbb{E}^{d}$ that have no point in common. Let $\Psi$ be the plane of least dimension $d$ that contains both $\Pi_{1}$ and $\Pi_{2}$. Let $r=p+q-d$. Then $\Pi_{1}$ and $\Pi_{2}$ intersect in an $r$-plane at infinity and we say that $\Pi_{1}$ and $\Pi_{2}$ are $(r+1) / q$ parallel.

If $p=q$ and $r=p-1$, then $d=p+1$, and $\Pi_{1}$ and $\Pi_{2}$ are contained in the $(p+1)$-plane $\Psi$. We say that $\Pi_{1}$ and $\Pi_{2}$ are completely parallel. When this occurs, then through each point $O$ in $\Psi$ there is a unique line in $\Psi$ that is normal to both $\Pi_{1}$ and $\Pi_{2}$. If two normals are drawn through two points $O, O^{\prime}$, cutting $\Pi_{1}$ and $\Pi_{2}$ in $A, B$ and $A^{\prime}, B^{\prime}$ then $A B B^{\prime} A^{\prime}$ is a rectangle and $A B=A^{\prime} B^{\prime}$. The distance $A B$ is called the distance between the completely parallel $p$-planes.

We deduce that if two completely parallel $p$-planes share a common point, then they are in fact the same $p$-plane.

In contrast to complete parallelism, we again refer to [19] in order that we may clarify complete orthogonality in higher dimensions.

DEFINITION (systems of $d$ mutually orthogonal lines). Through any point $O$ in $\mathbb{E}^{d}$ we can find $d$ lines that are all mutually perpendicular. We begin with a line 
$l_{1}$. All lines perpendicular to $l_{1}$ through $O$ form a $(d-1)$-plane $\Pi_{1}$ whose normal vector at $O$ is $l_{1}$. Let $l_{2}$ be one of these lines and let $\Pi_{2}$ be the $(d-1)$-plane whose normal vector at $O$ is $l_{2}$. Then all lines perpendicular to both $l_{1}$ and $l_{2}$ at $O$ lie in the $(d-2)$-plane that is the intersection of $\Pi_{1}$ and $\Pi_{2}$. Let $l_{3}$ be one of these lines. Continuing in this manner we create a system of $d$ lines $l_{1}, l_{2}, \ldots, l_{d}$ that are all mutually perpendicular. Any $p$ of these lines determine a $p$-plane $\Psi_{p}$, and the remaining $d-p$ lines determine a $(d-p)$-plane $\Psi_{d-p}$. These two planes only intersect at $O$ and have the property that every line of $\Psi_{p}$ through $O$ is perpendicular to every line of $\Psi_{d-p}$ through $O$. The two planes $\Psi_{p}$ and $\Psi_{d-p}$ are said to be completely orthogonal.

We deduce that for $\Psi_{p}$, defined as above and containing the point $O$, there exists a unique $(d-p)$-plane $\Psi_{d-p}$ that is completely orthogonal to $\Psi_{p}$ through $O$. Hence for a given system of $d$ mutually orthogonal lines in $\mathbb{E}^{d}$ and any point $O$, for each partition of the lines into two sets containing $p$ and $d-p$ lines there exists a unique pair of completely orthogonal planes, $\Psi_{p}$ and $\Psi_{d-p}$, that intersect only at $O$,

LEMMA 4.1. The number of integer points on 1-dimensional boundary components is estimated by

$$
\sum_{\operatorname{dim} S^{\star}\left(V_{i}\right)=1}\left|S^{\star}\left(V_{i}\right)\right| \leq \frac{2^{6 d-1} 3^{3} c_{1}^{(d-1) / 2} \pi^{d-1}}{\alpha_{d-1} c_{0}^{(d+1) / 2}} \delta\left(c_{1} M\right)^{d-1} .
$$

Proof. In the proof of Lemma 6.1 of [15] we noted that at most two one-dimensional boundary components can lie on the same straight line.

We consider all the boundary components $S^{\star}\left(V_{i}\right)$ which are 1-dimensional lying parallel to some primitive integer vector e. Suppose that the component contains $l$ points of $S(H)$, where

$$
L+1 \leq l \leq 2 L
$$

for some $L$ equal to a power of two. We can take $\mathbf{g}=(l-1) \mathbf{e}$ in Lemma 3.5, with

$$
|\mathbf{g}| \geq(l-1)|\mathbf{e}| \geq L|\mathbf{e}|
$$

In Lemma 3.5 the angle $\alpha$ between the vector $\mathbf{e}$ and the normal to $C_{1}$ at $T$, the point of $C_{1}$ nearest to $V$, satisfies

$$
|\cos \alpha| \leq \frac{52 \delta c_{1}}{c_{0} L|\mathbf{e}|} .
$$

Hence

$$
\left|\frac{\pi}{2}-\alpha\right| \leq \frac{26 c_{1} \pi \delta}{c_{0} L|\mathbf{e}|}
$$


We want to discuss the spacing of the vertices $V_{i}$ that label the enlarged components $S^{\prime}\left(V_{i}\right)$ and so the boundary components $S^{\star}\left(V_{i}\right)$. Each $V_{i}$ has a normal projection $T_{i}$ on $C_{1}$. Consider a $d$-dimensional sphere $B$ of radius $c_{1} M$. We map $T_{i}$ on $C_{1}$ to the point $W_{i}$ on $B$ where the outward normal $\mathbf{n}$ to $B$ is parallel to the outward normal to $C_{1}$ at $T_{i}$.

Let $V_{i}$ and $V_{j}$ be distinct vertices labelling enlarged vertex components. Since $V_{j} \notin S\left(V_{i}\right)$, we have

$$
T_{i} T_{j}>\sqrt{c_{0} \delta M}
$$

by (13) of Lemma 3.2. Since $C_{1}$ has sectional radii of curvature at most $c_{1} M$,

$$
W_{i} W_{j} \geq T_{i} T_{j}>\sqrt{c_{0} \delta M}
$$

Hence $d$-dimensional balls $B_{i}$, radii $\frac{1}{2} \sqrt{c_{0} \delta M}$, centred on the points $W_{i}$ on $B$, are disjoint.

The $d$-ball $B_{i}$ meets the surface of the $d$-sphere $B$ in a $(d-1)$-dimensional set $A_{i}$ which contains the centre $W_{i}$ of $B_{i}$ and is a $(d-1)$-ball in spherical geometry. As the $B_{i}$ are disjoint, the $(d-1)$-dimensional volumes of the sets $A_{i}$, on the boundary surface of the $d$-sphere $B_{i}$, are also disjoint and do not overlap. Hence different sets $S^{\prime}\left(V_{i}\right)$ correspond to disjoint sets $A_{i}$, centre $W_{i}$, on the surface of the $d$-sphere $B$. The $(d-1)$-volume of $A_{i}$ is greater than the $(d-1)$-volume of the intersection of a hyperplane through $W_{i}$ with $B_{i}$, which is

$$
\alpha_{d-1}\left(\sqrt{\frac{c_{0} \delta M}{4}}\right)^{d-1} .
$$

As $V_{i} \in S^{\star}\left(V_{i}\right)$ and $S^{\star}\left(V_{i}\right) \subseteq S^{\prime}\left(V_{i}\right)$, different sets $S^{\star}\left(V_{i}\right)$ also correspond to disjoint sets $A_{i}$, centre $W_{i}$, on the surface of the sphere $B$.

For each vector e, there is an equatorial hyperplane of the $d$-sphere $B$ at right angles to e. By (29) the point $W$ on the surface of $B$, where the normal is parallel to the normal $\mathbf{n}$ to $C_{1}$ at $T$ lies

$$
\leq \frac{26 \pi \delta c_{1} M}{c_{0} L|\mathbf{e}|}
$$

from the equatorial hyperplane measured along the surface of $B$. As stated, the set $A_{i}$ is the intersection of the surface of $B$ with a $d$-ball radius $\frac{1}{2} \sqrt{c_{0} \delta M}$, so it forms a $(d-1)$-ball in the spherical geometry of the surface of $B$, whose radius in spherical geometry is

$$
\begin{gathered}
\leq \frac{\pi}{2} \cdot \sqrt{\frac{c_{0} \delta M}{4}} \leq \pi \sqrt{\frac{c_{0} \delta M}{16}} \cdot \frac{4 \sqrt{\delta c_{1} M}}{L|\mathbf{e}|} \\
=\frac{\pi \delta c_{1} M}{L|\mathbf{e}|}\left(\frac{c_{0}}{c_{1}}\right)^{\frac{1}{2}} \leq \frac{\pi \delta c_{1} M}{c_{0} L|\mathbf{e}|}
\end{gathered}
$$


by (4) and (11).

Hence, each point of $A_{i}$ lies within a distance

$$
\leq \frac{26 \pi \delta c_{1} M}{c_{0} L|\mathbf{e}|}+\frac{\pi \delta c_{1} M}{c_{0} L|\mathbf{e}|}=\frac{27 \pi \delta c_{1} M}{c_{0} L|\mathbf{e}|}
$$

from the equatorial hyperplane, measured along the surface of the $d$-sphere $B$.

We consider the "girdle" of one-dimensional boundary components $S^{\star}\left(V_{i}\right)$ which are parallel to the fixed vector $\mathbf{e}$. The components in the girdle satisfying (28) correspond to points $W_{i}$ and sets $A_{i}$ on the surface of $B$, such that every point of $A_{i}$ lies close to the equatorial hyperplane perpendicular to e. The sets $A_{i}$ lie in a $(d-1)$-annulus whose volume in spherical geometry is at most

$$
\left(2 \pi c_{1} M\right)^{d-2}\left(\frac{54 \pi \delta c_{1} M}{c_{0} L|\mathbf{e}|}\right)=\frac{27(2 \pi)^{d-1} \delta\left(c_{1} M\right)^{d-1}}{c_{0} L|\mathbf{e}|}
$$

By (30) the number of disjoint sets $A_{i}$ in the girdle is at most

$$
\begin{gathered}
\frac{2^{d-1}}{\alpha_{d-1}\left(c_{0} \delta M\right)^{(d-1) / 2}} \cdot \frac{27(2 \pi)^{d-1} \delta\left(c_{1} M\right)^{d-1}}{c_{0} L|\mathbf{e}|} \\
=\frac{27\left(4 \pi c_{1}\right)^{d-1} M^{d-1 / 2}}{\alpha_{d-1} c_{0}^{(d+1) / 2} \delta^{(d-3) / 2} L|\mathbf{e}|}
\end{gathered}
$$

so the boundary components $S^{\star}\left(V_{i}\right)$ in the girdle for which the number $l$ of points is in the range (28) contribute at most

$$
\frac{54\left(4 \pi c_{1}\right)^{d-1} M^{(d-1) / 2}}{\alpha_{d-1} c_{0}^{(d+1) / 2} \delta^{(d-3) / 2}|\mathbf{e}|}
$$

integer points. The estimate (32) refers only to components in the girdle for which $l$ lies in the range (28). We keep the condition (28), and sum over primitive integer vectors e. Since the component is a straight line segment lying within the strip $E$, by (11) we have

$$
L|\mathbf{e}| \leq(l-1)|\mathbf{e}| \leq 4 \sqrt{\delta c_{1} M} .
$$

We note that if two boundary components lie on the same line, then the vertices $V_{i}$ which label the boundary components $S^{\star}\left(V_{i}\right)$ must be different, so they are counted separately in this argument. We use the bound of Lemma 3.6 with $j=1$ to sum over $\mathbf{e}$, so that the number of points on one-dimensional boundary components with $l$ in the range (28) is at most

$$
\begin{gathered}
\frac{54\left(4 \pi c_{1}\right)^{d-1} M^{(d-1) / 2}}{\alpha_{d-1} c_{0}^{(d+1) / 2} \delta^{(d-3) / 2}} \cdot 2^{2 d+1}\left(\frac{4 \sqrt{\delta c_{1} M}}{L}\right)^{d-1} \\
=\frac{2^{6 d-2} 3^{3} c_{1}^{(d-1) / 2} \pi^{d-1} \delta\left(c_{1} M\right)^{d-1}}{\alpha_{d-1} c_{0}^{(d+1) / 2} L^{d-1}} .
\end{gathered}
$$


Finally we remove the condition (28) by summing $L$ through powers of 2 , noting that

$$
\left(1+\frac{1}{2^{k}}+\frac{1}{4^{k}}+\frac{1}{8^{k}}+\ldots\right) \leq \frac{2^{k}}{2^{k}-1} \leq 2 .
$$

Hence the total number of integer points of $S(H)$ which lie on one-dimensional boundary components is at most

$$
\left(\frac{2^{6 d-1} 3^{3} c_{1}^{(d-1) / 2} \pi^{d-1}}{\alpha_{d-1} c_{0}^{(d+1) / 2}}\right) \delta\left(c_{1} M\right)^{d-1} .
$$

LEMMA 4.2. The number of integer points on $(d-1)$-dimensional boundary components, when $\delta \leq \delta_{0}$, is estimated by

$$
\begin{gathered}
\sum_{\operatorname{dim} S^{\star}\left(V_{i}\right)=d-1}\left|S^{\star}\left(V_{i}\right)\right| \\
\leq d !(d+1) ! 2^{\frac{9 d+17}{2}}\left(\frac{c_{1}}{c_{0}}\right)^{(d-1) / 2}\left(\left(c_{1} M\right)^{\frac{d(d-1)}{d+1}}+2\left(\frac{c_{1}}{c_{0}}\right)^{(d-1) / 2} \delta_{0}\left(c_{1} M\right)^{d-1}\right) .
\end{gathered}
$$

Proof. Each $(d-1)$-dimensional boundary component $S^{\star}\left(V_{i}\right)$ is part of a hyperplane. The intersection of all such hyperplanes forms a convex polytope, $H^{\star}$, that is contained within the convex hull $H$ and the vertices of $H^{\star}$ are points of $S(H)$. Let $\Psi$ be a hyperplane face of $H^{\star}$, with outward normal vector $\mathbf{n}$ with respect to $H^{\star}$ (a primitive integer vector). Let $Z$ be the point of $C$ at which the normal $\mathbf{m}$ to $C$ is parallel to $\mathbf{n}$, with $\mathbf{n}$ as outward normal vector. Let $\mathbf{m}$ cut $\Psi$ in $Y$ and the boundary surfaces $C_{0}$ and $C_{1}$ in $W$ and $X$ respectively (Figure 1 ). Then $\mathbf{m}$ is also the outward normal to $C_{0}$ at $W$, to $C_{1}$ at $X$, and the boundary hyperplane $\Psi$ of the convex hull $H^{\star}$ at $Y$. Let $h=X Y, h^{\prime}=W Y$ be the heights of $X$ above $\Psi$ and of $W$ above or below $\Psi$ as depicted in Figure 1. Each component in the annulus $E \cap \Pi$ is convex. We apply Lemma 2.2 with $j=d-1$. The set of points is strictly $(d-1)$-dimensional so we use the minor arc case of Lemma 2.2 with $j=d-1$, and lattice determinant $n=|\mathbf{n}|$ by Lemma 2.1. The volume $V$ is estimated in Lemma 3.11, so we have an estimate for the number of integer points $N(\Psi)$ that lie in $E \cap \Psi$ such that

$$
\begin{gathered}
N(\Psi) \leq \frac{(d-1) ! V}{|\mathbf{n}|}+d-1 \leq \frac{d ! V}{|\mathbf{n}|} \\
\leq \frac{d ! 2^{\frac{d+9}{2}} d \delta\left(c_{1} M\right)^{\frac{d-1}{2}} h^{\frac{d-3}{2}}}{|\mathbf{n}|} .
\end{gathered}
$$

We sum over all the outward normal vectors of the hyperplanes $\Psi$. We get the total number of integer points on the $(d-1)$-boundary components, $N$, to be

$$
N \leq \sum N(\Psi) \leq d ! 2^{\frac{d+9}{2}} d \delta\left(c_{1} M\right)^{\frac{d-1}{2}} h^{\frac{d-3}{2}} \sum \frac{1}{|\mathbf{n}|}
$$




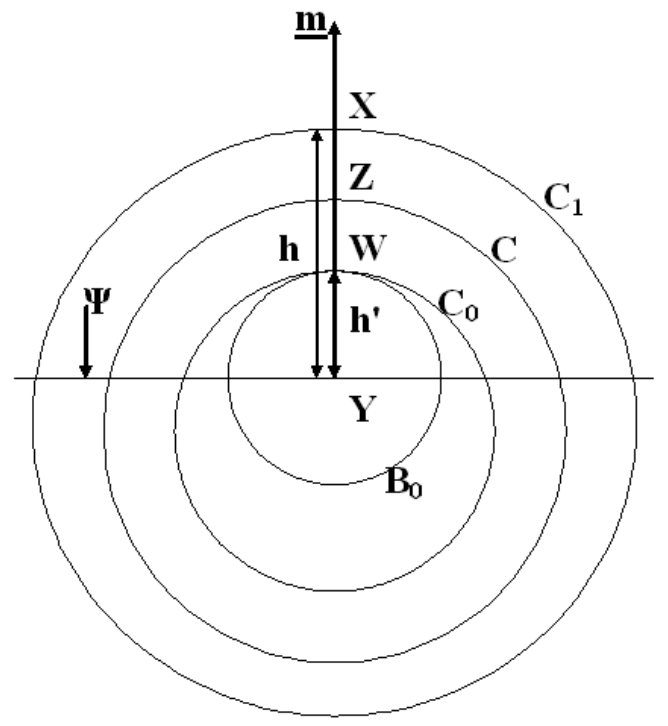

Figure 1: Heights along the common normal $\ell$.

We distinguish various cases according to the order of the points $W, X, Y$ and $Z$ on the normal $l$. If $h>2 \delta$ then the point $W$ lies between $X$ and $Y$ and $h^{\prime}>0$, as shown in Figure 1. By the Curvature Condition, a $d$-ball $B_{0}$ of radius $c_{0} M$, touching $C_{0}$ at $W$, fits completely inside $C_{0}$. Since $h^{\prime}>0$, the hyperplane $\Psi$ cuts both $C_{0}$ and $B_{0}$. A "cap" of the hypersurface $C_{0}$ lies above the hyperplane $\Psi$. The $(d-1)$-dimensional surface content $A$ of the cap cut from $C_{0}$ is greater than the content $A^{\prime}$ of its projection onto the plane $\Psi$. If $h \leq c_{0} M+2 \delta$, then the equator of the $d$-ball $B_{0}$ lies below $\Psi$, and $A^{\prime} \geq A^{\prime \prime}$, the $(d-1)$-dimensional content of $B_{0} \cap \Psi$. This was calculated in the proof in [15] of our Lemma 3.11, so we have

$$
A \geq A^{\prime} \geq A^{\prime \prime}=\alpha_{d-1}\left(\left(2 c_{0} M-h^{\prime}\right) h^{\prime}\right)^{\frac{d-1}{2}} .
$$

For given $h_{0} \geq 4 \delta$, let $Q\left(h_{0}\right)$ be the number of hyperplane faces of $H$ with height in the range $h \geq h_{0}$. Let $h_{0}^{\prime}=h_{0}-2 \delta(\geq 2 \delta)$.

First we consider the extreme case

$$
h \geq c_{0} M+2 \delta .
$$

The equatorial plane $\Psi^{\star}$ parallel to $\Psi$ through the centre of $B_{0}$, cuts off a cap from $C_{0}$ of smaller $(d-1)$-dimensional content $A^{\star}$. Then $A^{\star}$ is greater than or equal to half the surface content of the ball $B_{0}$, which is greater than $B_{0} \cap \Psi^{\star}$, so that

$$
A \geq A^{\star} \geq \frac{1}{2} d \alpha_{d}\left(c_{0} M\right)^{d-1} \geq B_{0} \cap \Psi^{\star}=\alpha_{d-1}\left(c_{0} M\right)^{d-1} .
$$

The boundary content of $C_{0}$ is less than or equal to that of a $d$-sphere radius 
$c_{1} M$

$$
\leq d \alpha_{d}\left(c_{1} M\right)^{d-1}
$$

Let $Q_{E}$ be the number of 'extreme faces' satisfying (38). Dividing the upper bound (40) by the lower bound (39) gives

$$
Q_{E} \leq \frac{d \alpha_{d}\left(c_{1} M\right)^{d-1}}{\alpha_{d-1}\left(c_{0} M\right)^{d-1}}=\frac{d \alpha_{d}}{\alpha_{d-1}}\left(\frac{c_{1}}{c_{0}}\right)^{d-1}=\lambda_{E}
$$

say.

Secondly we consider the usual case

$$
h \leq c_{0} M+2 \delta
$$

so that $h_{0}^{\prime}=h_{0}-2 \delta \leq h-2 \delta \leq c_{0} M$. Then from (37)

$$
A \geq \alpha_{d-1}\left(\left(2 c_{0} M-h^{\prime}\right) h^{\prime}\right)^{\frac{d-1}{2}} \geq \alpha_{d-1}\left(\left(2 c_{0} M-h_{0}^{\prime}\right) h_{0}^{\prime}\right)^{\frac{d-1}{2}} .
$$

Let $Q_{U}\left(h_{0}\right)$ be the number of 'usual' faces with height $h \geq h_{0}$ satisfying (42). Dividing the upper bound, (40), by the lower bound, (43) for this case gives

$$
Q_{U}\left(h_{0}\right) \leq \frac{d \alpha_{d}\left(c_{1} M\right)^{d-1}}{\alpha_{d-1}\left(\left(2 c_{0} M-h_{0}^{\prime}\right) h_{0}^{\prime}\right)^{\frac{d-1}{2}}} .
$$

We simplify the upper bound (44). When $4 \delta \leq h_{0} \leq c_{0} M+2 \delta$, then $2 \delta \leq h_{0}^{\prime} \leq$ $c_{0} M$. This implies that

$$
\frac{1}{2 c_{0} M-h_{0}^{\prime}}=\frac{1}{2 c_{0} M-h_{0}+2 \delta} \leq \frac{1}{c_{0} M}
$$

and

$$
\frac{1}{h_{0}^{\prime}} \leq \frac{2}{h_{0}}
$$

Hence we can write

$$
\begin{gathered}
Q_{U}\left(h_{0}\right) \leq \frac{2^{\frac{d-1}{2}} d \alpha_{d}\left(c_{1} M\right)^{d-1}}{\alpha_{d-1}\left(c_{0} M h_{0}\right)^{\frac{d-1}{2}}} \\
\leq \frac{d \alpha_{d}}{\alpha_{d-1}}\left(\frac{c_{1}}{c_{0}}\right)^{\frac{d-1}{2}}\left(\frac{2 c_{1} M}{h_{0}}\right)^{\frac{d-1}{2}}=\lambda_{U}\left(\frac{2 c_{1} M}{h_{0}}\right)^{\frac{d-1}{2}}
\end{gathered}
$$

say.

Each face $\Psi$ is contained within the outer shell boundary $C_{1}$, which itself is contained within a $d$-hypersphere of radius $c_{1} M$. Therefore all heights are at most $2 c_{1} M$, and we have

$$
Q\left(h_{0}\right) \leq Q_{U}\left(h_{0}\right)+Q_{E}
$$




$$
\begin{gathered}
\leq\left(\lambda_{E}+\lambda_{U}\right)\left(\frac{2 c_{1} M}{h_{0}}\right)^{\frac{d-1}{2}} \leq\left(\frac{2 d \alpha_{d}}{\alpha_{d-1}}\right)\left(\frac{\sqrt{2} c_{1}}{c_{0}}\right)^{d-1}\left(\frac{c_{1} M}{h_{0}}\right)^{\frac{d-1}{2}} \\
\leq 2^{\frac{d+5}{2}} d\left(\frac{c_{1}}{c_{0}}\right)^{d-1}\left(\frac{c_{1} M}{h_{0}}\right)^{\frac{d-1}{2}}=\lambda_{1}\left(\frac{c_{1} M}{h_{0}}\right)^{\frac{d-1}{2}},
\end{gathered}
$$

say, where we have used (7). This result is valid for all faces with height $h \geq$ $h_{0} \geq 4 \delta$.

For a fixed height $h_{0}$, the sum in (36) is maximal when as many short vectors as possible are counted, up to the upper bound in (46). In the proof of Lemma 3.6 we saw that there are at most $2^{2 d-1} F^{d}$ vectors in each of the partitions and the inequality (20) is calculated assuming this maximum.

The total number of faces counted is

$$
\begin{gathered}
2^{2 d-1}\left(\left(2^{0}\right)^{d}+\left(2^{1}\right)^{d}+\left(2^{2}\right)^{d}+\ldots+\left(2^{k}\right)^{d}\right)=2^{2 d-1} \frac{\left(\left(2^{d}\right)^{k+1}-1\right)}{2^{d}-1} \\
\geq 2^{d(k+1)+d-1} \geq 2^{d(k+1)} .
\end{gathered}
$$

Therefore, to ensure that all possible faces are counted, we require

$$
2^{d(k+1)} \geq \lambda_{1}\left(\frac{c_{1} M}{h_{0}}\right)^{\frac{d-1}{2}}
$$

which implies that

$$
2^{d k} \geq \frac{\lambda_{1}}{2^{d}}\left(\frac{c_{1} M}{h_{0}}\right)^{\frac{d-1}{2}}
$$

Hence if

$$
E=\lambda_{1}^{\frac{1}{d}}\left(\frac{c_{1} M}{h_{0}}\right)^{\frac{d-1}{2 d}} \geq 2^{k} \geq\left(\frac{\lambda_{1}}{2^{d}}\right)^{\frac{1}{d}}\left(\frac{c_{1} M}{h_{0}}\right)^{\frac{d-1}{2 d}}
$$

in Lemma 3.6 with $j=1$, then (36) is maximal. We have

$$
\sum_{1 \leq|\mathbf{e}| \leq 2^{k}} \frac{1}{|\mathbf{e}|} \leq 2^{2 d+1}\left(\lambda_{1}^{\frac{1}{d}}\left(\frac{c_{1} M}{h_{0}}\right)^{\frac{d-1}{2 d}}\right)^{d-1} .
$$

We now consider three cases.

Case 1.

$$
h \geq \frac{1}{\left(c_{1} M\right)^{\frac{d-1}{d+1}}} \geq 4 \delta .
$$

Let $L$ be the total number of $(d-1)$-faces satisfying (49). We partition these $(d-1)$-faces into sets $G_{1}, G_{2}, \ldots, G_{n}$, according to their respective heights $h_{i}, 1 \leq$ $i \leq n$, where $h_{n}>h_{n-1}>\ldots>h_{2}>h_{1} \geq 4 \delta$. Let $L_{i}=\left|G_{i}\right|$, the number of 
hyperplane faces whose height is $h_{i}$; let $\mathbf{n}_{i, 1}, \mathbf{n}_{i, 2}, \ldots, \mathbf{n}_{i, L_{i}}$ be the normal vectors of the faces in $G_{i}$ and let

$$
\sigma_{i}=\sum_{j=1}^{L_{i}} \frac{1}{\left|\mathbf{n}_{i, j}\right|}
$$

By (47) we have

$$
\sum_{i=1}^{n} \sigma_{i} \leq \sum_{1 \leq|\mathbf{e}| \leq 2^{k}} \frac{1}{|\mathbf{e}|} \leq 2^{2 d+1}\left(\lambda_{1}^{\frac{1}{d}}\left(\frac{c_{1} M}{h_{i}}\right)^{\frac{d-1}{2 d}}\right)^{d-1}
$$

Hence for each $h_{i}$, there exists a real number $\tau_{i}, 0<\tau \leq 1$ with

$$
\sigma_{i}=\tau_{i} 2^{2 d+1}\left(\lambda_{1}^{\frac{1}{d}}\left(\frac{c_{1} M}{h_{i}}\right)^{\frac{d-1}{2 d}}\right)^{d-1}
$$

and

$$
0<\sum_{i=1}^{n} \tau_{i} \leq 1
$$

Let $N\left(h_{i}\right)$ be the number of integer points lying in $G_{i} \cap E$. Then by (35) and (51), we have

$$
\begin{gathered}
N\left(h_{i}\right) \leq d ! 2^{\frac{d+9}{2}} d \delta\left(c_{1} M\right)^{\frac{d-1}{2}} h_{i}^{\frac{d-3}{2}} \sum_{j=1}^{L_{i}} \frac{1}{\left|\mathbf{n}_{i, j}\right|} \\
\leq d ! 2^{\frac{d+9}{2}} d \delta\left(c_{1} M\right)^{\frac{d-1}{2}} h_{i}^{\frac{d-3}{2}} \tau_{i} 2^{2 d+1}\left(\lambda_{1}^{\frac{1}{d}}\left(\frac{c_{1} M}{h_{i}}\right)^{\frac{d-1}{2 d}}\right)^{d-1} . \\
=\lambda_{2} \tau_{i} \delta\left(c_{1} M\right)^{\frac{d-1}{2}}+\frac{(d-1)^{2}}{2 d} h_{i}^{\frac{d-3}{2}}-\frac{(d-1)^{2}}{2 d}
\end{gathered}
$$

say. Summing over all heights $h_{i}$ gives $N_{1}$, the total number of integer points contributed in this case to be

$$
\leq \lambda_{2} \delta\left(c_{1} M\right)^{\frac{(d-1)(2 d-1)}{2 d}} \sum_{i=1}^{n} \tau_{i} h_{i}^{\frac{-(d+1)}{2 d}} .
$$

The exponent of $h_{i}$ in (53) is negative, and as the $h_{i}$ are positive, the sum is maximal when the $h_{i}$ are as small as possible and the $\tau_{i}$ are as large as possible for the smallest $h_{i}$. Hence we take

$$
\sum_{i=1}^{n} \tau_{i}=1
$$

in $(53)$, and

$$
h_{i}=\frac{1}{\left(c_{1} M\right)^{\frac{d-1}{d+1}}}
$$


for all $i$. Substituting for $h_{i}$ in (53) gives the total number of integer points $N_{1}$ contributed to be

$$
N_{1} \leq \lambda_{2} \delta\left(c_{1} M\right)^{\frac{(d-1)(2 d-1)}{2 d}}+\frac{d-1}{2 d} \sum_{i=1}^{n} \tau_{i}=\lambda_{2} \delta\left(c_{1} M\right)^{d-1} .
$$

Case 2.

$$
4 \delta \leq h \leq \frac{1}{\left(c_{1} M\right)^{\frac{d-1}{d+1}}} .
$$

By Lemma 3.9, the maximum possible number of faces is

$$
\leq 2\left(3 \alpha_{d} d !\right)^{\frac{d}{d+1}}\left(c_{1} M\right)^{\frac{d(d-1)}{d+1}} .
$$

Hence if

$$
E=4\left(3 \alpha_{d} d !\right)^{\frac{1}{d+1}}\left(c_{1} M\right)^{\frac{d-1}{d+1}} \geq 2^{k} \geq 2\left(3 \alpha_{d} d !\right)^{\frac{1}{d+1}}\left(c_{1} M\right)^{\frac{d-1}{d+1}}
$$

in Lemma 3.6 with $j=1$, then (36) is maximal. We have

$$
\sum_{1 \leq|\mathbf{e}| \leq 2^{k}} \frac{1}{|\mathbf{e}|} \leq 2^{2 d+1}\left(4\left(3 \alpha_{d} d !\right)^{\frac{1}{d+1}}\left(c_{1} M\right)^{\frac{d-1}{d+1}}\right)^{d-1} .
$$

Let $N_{2}$ be the total number of integer points in this case. Then substituting (56) into (36) yields

$$
N_{2} \leq d ! 2^{\frac{d+9}{2}} d \delta\left(c_{1} M\right)^{\frac{d-1}{2}} h^{\frac{d-3}{2}} \cdot 2^{2 d+1} 4^{d-1}\left(3 \alpha_{d} d !\right)^{\frac{d-1}{d+1}}\left(c_{1} M\right)^{\frac{(d-1)^{2}}{d+1}} .
$$

Taking

$$
h=\frac{1}{\left(c_{1} M\right)^{\frac{d-1}{d+1}}}
$$

to maximise (57) we have

$$
N_{2} \leq \lambda_{3} \delta\left(c_{1} M\right)^{\frac{(d-1)^{2}}{d+1}-\frac{(d-3)(d-1)}{2(d+1)}+\frac{d-1}{2}}=\lambda_{3} \delta\left(c_{1} M\right)^{d-1} .
$$

Case 3. $0 \leq h \leq 4 \delta$. As in the previous case, we assume the maximum number of short vector faces and we take $h=4 \delta$ to maximise (57). Let $N_{3}$ be the total number of integer points in this case. Then

$$
\begin{gathered}
N_{3} \leq \lambda_{3} \delta(4 \delta)^{\frac{d-3}{2}}\left(c_{1} M\right)^{\frac{(d-1)^{2}}{d+1}}+\frac{d-1}{2} \\
=\lambda_{3} 4^{\frac{d-3}{2}}\left(\delta c_{1} M\right)^{\frac{d-1}{2}}\left(c_{1} M\right)^{\frac{(d-1)^{2}}{d+1}} .
\end{gathered}
$$


When

$$
\delta \leq \delta_{0}=\left(\frac{c_{0}}{2^{2 d} 5^{d-1} 13 d ! c_{1}}\right)^{\frac{2}{d+1}}\left(c_{1} M\right)^{\frac{-(d-1)}{d+1}}=\mu\left(c_{1} M\right)^{\frac{-(d-1)}{d+1}}
$$

then we have the bound

$$
N_{3} \leq \lambda_{3} \mu^{\frac{(d-1)}{2}} 2^{d-3}\left(\left(c_{1} M\right)^{\frac{2}{d+1}}\right)^{\frac{d-1}{2}}\left(c_{1} M\right)^{\frac{(d-1)^{2}}{d+1}}=\lambda_{3} \mu^{\frac{(d-1)}{2}} 2^{d-3}\left(c_{1} M\right)^{\frac{d(d-1)}{d+1}} .
$$

Finally we add together the upper bounds for $N_{1}, N_{2}$ and $N_{3}$ in (54), (58) and (59) respectively. When $\delta=\delta_{0}$ this gives the total number of integer points lying on the $(d-1)$-dimensional boundary components, $N$, to be

$$
N \leq\left(\lambda_{2}+\lambda_{3}\right) \delta_{0}\left(c_{1} M\right)^{d-1}+\lambda_{3} \mu^{\frac{d-1}{2}} 2^{d-3}\left(c_{1} M\right)^{\frac{d(d-1)}{d+1}} .
$$

After simplification we find that

$$
\begin{gathered}
\lambda_{2} \leq d(d+1) ! 2^{3 d+8}\left(\frac{c_{1}}{c_{0}}\right)^{d-1}, \\
\lambda_{3} \leq d !(d+1) ! 2^{\frac{9 d+17}{2}},
\end{gathered}
$$

and

$$
2^{d-3} \mu^{\frac{d-1}{2}} \leq 1
$$

where we have used (6). Hence, if $\delta \leq \delta_{0}$ then $N$

$$
\leq d !(d+1) ! 2^{\frac{9 d+17}{2}}\left(\frac{c_{1}}{c_{0}}\right)^{(d-1) / 2}\left(\left(c_{1} M\right)^{\frac{d(d-1)}{d+1}}+2\left(\frac{c_{1}}{c_{0}}\right)^{(d-1) / 2} \delta_{0}\left(c_{1} M\right)^{d-1}\right) .
$$

LEMMA 4.3. The number of integer points on d-dimensional boundary components, when $\delta=\delta_{0}$, is estimated by

$$
\begin{gathered}
\sum_{\operatorname{dim} S^{\star}\left(V_{i}\right)=d}\left|S^{\star}\left(V_{i}\right)\right| \leq 2(d+1)\left(3 \alpha_{d} d !\right)^{\frac{d}{d+1}}\left(2 c_{1} M\right)^{\frac{d(d-1)}{d+1}} \\
\leq 36(d+1) !\left(2 c_{1} M\right)^{\frac{d(d-1)}{d+1}}
\end{gathered}
$$

Proof. From (18), the $d$-dimensional boundary component $S^{\star}\left(V_{i}\right)$ will have a $d$ dimensional volume $\operatorname{Vol}\left(H_{i}\right)$, with

$$
\operatorname{Vol}\left(H_{i}\right) \leq \frac{52 \delta c_{1}}{c_{0}}\left(20 \sqrt{\delta c_{1} M}\right)^{d-1}
$$


Since $\delta=\delta_{0}$ this gives a $d$-volume of at most $1 / d$ !. Applying the minor arc case of Lemma 2.2 the gives

$$
K_{i} \leq(d+1) ! \operatorname{Vol}\left(H_{i}\right),
$$

where $K_{i}$ is the number of integer points contained in $S^{\star}\left(V_{i}\right)$. However, the existence of a $d$-dimensional $S^{\star}\left(V_{i}\right)$ in $S^{\prime}(V)$ requires that $K_{i} \geq d+1$, and so if we consider $\delta=\delta_{0}$, then $K_{i}$, the number of integer points in the boundary component, is exactly $d+1$. The number of vertices of the convex hull is

$$
\leq 2\left(3 \alpha_{d} d !\right)^{\frac{d}{d+1}}\left(2 c_{1} M\right)^{\frac{d(d-1)}{d+1}},
$$

by (25) in Lemma 3.10 with $j=1$. Hence, when $\delta=\delta_{0}$, the total number of integer points in the $d$-dimensional boundary components is estimated by

$$
\leq 2(d+1)\left(3 \alpha_{d} d !\right)^{\frac{d}{d+1}}\left(2 c_{1} M\right)^{\frac{d(d-1)}{d+1}} .
$$

\section{Girdles and Lattice Determinants}

We now recall Minkowski's Second Theorem [7].

LEMMA 5.1 (Minkowski's Second Theorem). Let $K$ be a convex body symmetrical in the origin. Let $\Lambda$ be a lattice. Let the successive minima of $K$ with respect to $\Lambda$ be $\lambda_{1}, \lambda_{2}, \ldots, \lambda_{d}$, defined by

$\lambda_{i}=\inf \{\lambda>0: \lambda$ K contains at least $i$ linearly independent vectors of $\Lambda\}$,

where

$$
0<\lambda_{1} \leq \lambda_{2} \leq \ldots \leq \lambda_{d}<+\infty
$$

Then they obey the inequality

$$
\frac{2^{d} D(\Lambda)}{d !} \leq \lambda_{1} \lambda_{2} \lambda_{3} \ldots \lambda_{d} V(K) \leq 2^{d} D(\Lambda)
$$

where $V(K)$ is the volume of $K$ and $D(\Lambda)$ is the determinant of the lattice.

COROLLARY. Let $\Lambda$ and $D(\Lambda)$ be defined as above, with $\lambda_{1}, \ldots, \lambda_{d}$ the ordinary Euclidean lengths of the lattice vectors. Let $K$ be the open unit d-ball, then the determinant or fundamental volume of the lattice satisfies

$$
\frac{\lambda_{1} \lambda_{2} \lambda_{3} \ldots \lambda_{d} \alpha_{d}}{2^{d}} \leq D(\Lambda) \leq \lambda_{1} \lambda_{2} \lambda_{3} \ldots \lambda_{d} .
$$


Proof of Corollary. By construction, if $\mathbf{e}_{1}, \mathbf{e}_{2}, \ldots, \mathbf{e}_{d}$ are the basis vectors of $\Lambda$ with respective Euclidean lengths $\lambda_{1}, \lambda_{2}, \ldots \lambda_{d}$, then the $\mathbf{e}_{i}$ are ordered by length. Let $\theta_{i}$ be the angle between $\mathbf{e}_{i+1}$ and the $i$-dimensional plane lattice defined by $\mathbf{e}_{1}, \mathbf{e}_{2}, \ldots, \mathbf{e}_{i}$ with determinant $D\left(\Lambda_{i}\right)$. Then

$$
\begin{gathered}
D(\Lambda)=\lambda_{d} \sin \theta_{d-1} D\left(\Lambda_{d-1}\right)=\lambda_{d} \lambda_{d-1} \sin \theta_{d-1} \sin \theta_{d-2} D\left(\Lambda_{d-2}\right)=\ldots \\
\ldots=\lambda_{1} \lambda_{2} \lambda_{3} \ldots \lambda_{d} \prod_{i=1}^{d} \sin \theta_{i} \leq \lambda_{1} \lambda_{2} \lambda_{3} \ldots \lambda_{d}
\end{gathered}
$$

The upper bound of (62) gives

$$
\frac{\lambda_{1} \lambda_{2} \lambda_{3} \ldots \lambda_{d} V(K)}{2^{n}} \leq D(\Lambda)
$$

and taking $V(K)=\alpha_{d}$ gives the required result.

Here we introduce the idea of a $j$-dimensional girdle, $2 \leq j \leq d-2$, with fixed basis vectors $\mathbf{e}_{1}, \mathbf{e}_{2}, \mathbf{e}_{3}, \ldots, \mathbf{e}_{j}$. The vectors $\mathbf{e}_{1}, \mathbf{e}_{2}, \mathbf{e}_{3}, \ldots, \mathbf{e}_{j}$ through the origin generate a $j$-dimensional lattice $\Lambda$ in a $j$-plane $\Pi_{0}$. Each $j$-girdle is therefore defined to be a set of $j$-dimensional boundary components whose $j$-planes $\Pi$ are all completely parallel to $\Pi_{0}$. The sets of integer points on each $j$-plane $\Pi$ are cosets of $\Lambda$, congruent to $\Lambda$ by translation, and the number of integer points lying on each $j$-girdle is related to the fundamental $j$-volume or determinant of the lattice $\Lambda$. Conversely the lattice $\Lambda$ determines the minimal basis $\mathbf{e}_{1}, \mathbf{e}_{2}, \mathbf{e}_{3}, \ldots, \mathbf{e}_{j}$ in the Corollary to Lemma 5.1. We write $l(\Lambda)$ for the length $\lambda_{j}$ of the longest basis vector $\mathbf{e}_{j}$ and introduce the following lemma to assist with our counting argument.

LEMMA 5.2 (sums of reciprocal lattice determinants). For $k=1,2, \ldots, d-1$ we have

$$
\sum_{l(\Lambda) \leq E} \frac{1}{(D(\Lambda))^{k}} \leq \frac{\left(2^{2 d+2 k} E^{d-k}\right)^{j}}{\alpha_{j}^{k}},
$$

where the sum ranges over all possible $j$-dimensional lattice determinants, $j \leq$ $d-1$, whose basis vectors have length $\leq E$. When we take $E$ to be the maximum possible length of a boundary component basis vector, then by (16), $E=10 \sqrt{\delta c_{1} M}$ and

$$
\sum_{l(\Lambda) \leq E} \frac{1}{(D(\Lambda))^{k}} \leq \frac{\left(2^{3 d+k}\left(5 \sqrt{\delta c_{1} M}\right)^{d-k}\right)^{j}}{\alpha_{j}^{k}} .
$$

Proof. By the Corollary to Lemma 5.1, there are basis vectors $\mathbf{e}_{i}, 1 \leq i \leq j$, of the lattice $\Lambda$ with

$$
\frac{\left|\mathbf{e}_{1}\right|\left|\mathbf{e}_{2}\right| \ldots\left|\mathbf{e}_{j}\right| \alpha_{j}}{2^{j}} \leq D(\Lambda) \leq\left|\mathbf{e}_{1}\right|\left|\mathbf{e}_{2}\right| \ldots\left|\mathbf{e}_{j}\right| .
$$


Hence by Lemma 5.1 and Lemma 3.6

$$
\begin{gathered}
\sum_{l(\Lambda) \leq E} \frac{1}{(D(\Lambda))^{k}} \leq\left(\frac{2^{j}}{\alpha_{j}}\right)^{k} \sum_{\left|\mathbf{e}_{1}\right| \leq E} \sum_{\left|\mathbf{e}_{2}\right| \leq E} \ldots \sum_{\left|\mathbf{e}_{j}\right| \leq E} \frac{1}{\left|\mathbf{e}_{1}\right|^{k}\left|\mathbf{e}_{2}\right|^{k} \ldots\left|\mathbf{e}_{j}\right|^{k}} \\
\leq\left(\frac{2^{j}}{\alpha_{j}}\right)^{k}\left(2^{2 d+k} E^{d-k}\right)^{j}=\frac{\left(2^{2 d+2 k} E^{d-k}\right)^{j}}{\alpha_{j}^{k}} .
\end{gathered}
$$

By (16) the vectors $\left|\mathbf{e}_{i}\right|$ are non-zero integer vectors with

$$
\left|\mathbf{e}_{i}\right| \leq l(\Lambda) \leq E=10 \sqrt{\delta c_{1} M}
$$

so that

$$
\sum_{l(\Lambda) \leq E} \frac{1}{(D(\Lambda))^{k}} \leq \frac{\left(2^{2 d+2 k}\left(10 \sqrt{\delta c_{1} M}\right)^{d-k}\right)^{j}}{\alpha_{j}^{k}}=\frac{\left(2^{3 d+k}\left(5 \sqrt{\delta c_{1} M}\right)^{d-k}\right)^{j}}{\alpha_{j}^{k}}
$$

which establishes the result.

\section{Summing the Boundary Components}

When we consider a $j$-dimensional boundary component $S^{\star}(V), 2 \leq j \leq d-$ 2 , there are geometrical considerations. The points of $S^{\star}(V)$ lie on some $j$ dimensional plane $\Pi$ containing the vertex $V$. The lattice of integer points meets $\Pi$ is some $j$-dimensional lattice $\Lambda$ with a basis consisting of $j$ integer vectors $\mathbf{e}_{1}, \mathbf{e}_{2}, \mathbf{e}_{3}, \ldots, \mathbf{e}_{j}$. The points of $S^{\star}(V)$ lie in the set $E$, the shell bounded by the surfaces $C_{1}$ and $C_{0}$. By the calculations of Lemma 3.3 the points of $S^{\star}(V)$ lie in a $d$-dimensional cylindrical slab $G$ whose axis is the normal $\mathbf{n}$ to $C_{1}$ at $R$, the point of $C_{1}$ closest to the vertex $V$. The upper and lower faces of the $d$-cylinder $G$ lie in the tangent hyperplane $F$ at $R$ and in a completely parallel hyperplane $F^{\prime}$, separated by a small distance

$$
\eta=\frac{52 \delta c_{1}}{c_{0}}
$$

The upper and lower faces of the $d$-cylinder are $(d-1)$-spheres of radius $10 \sqrt{\delta c_{1} M}$ by (16) of Lemma 3.3.

As defined at the beginning of section 4 , in $d$-dimensional space, through a given point $V$ on a $j$-plane $\Pi$, there exists a unique $(d-j)$-plane $\Psi$ that is completely orthogonal to $\Pi$.

Let $W_{1}$ be a point of $F^{\prime}$ not in $\Pi$ or $\Psi$ and lying at a distance $10 \sqrt{\delta c_{1} M}$ from the axis of the $d$-cylinder. As $2 \leq j, d-j \leq d-2$, we can choose $W_{1}$ such that $Y$, the (two-dimensional) affine plane defined by $\mathbf{n}$ and $W_{1}$, contains at least one other point $P$ of the $j$-plane $\Pi$ in addition to the vertex $V$. Then $Y \cap G$ is a 
rectangle containing $P, R$ and $V$, and $W_{1}$ is a corner of the rectangle. Hence the line segment $V P$ is also contained in $Y \cap \Pi$. Let $\mathbf{k}$ be the line $V P$ produced in $Y \cap \Pi$, cutting the hyperplanes of the upper and lower faces of the cylinder in $W_{3}$ and $W_{4}$. Let $W_{2}$ be the corner of the rectangle on $F$ that is diametrically opposite $W_{1}$ as depicted in Figure 2.

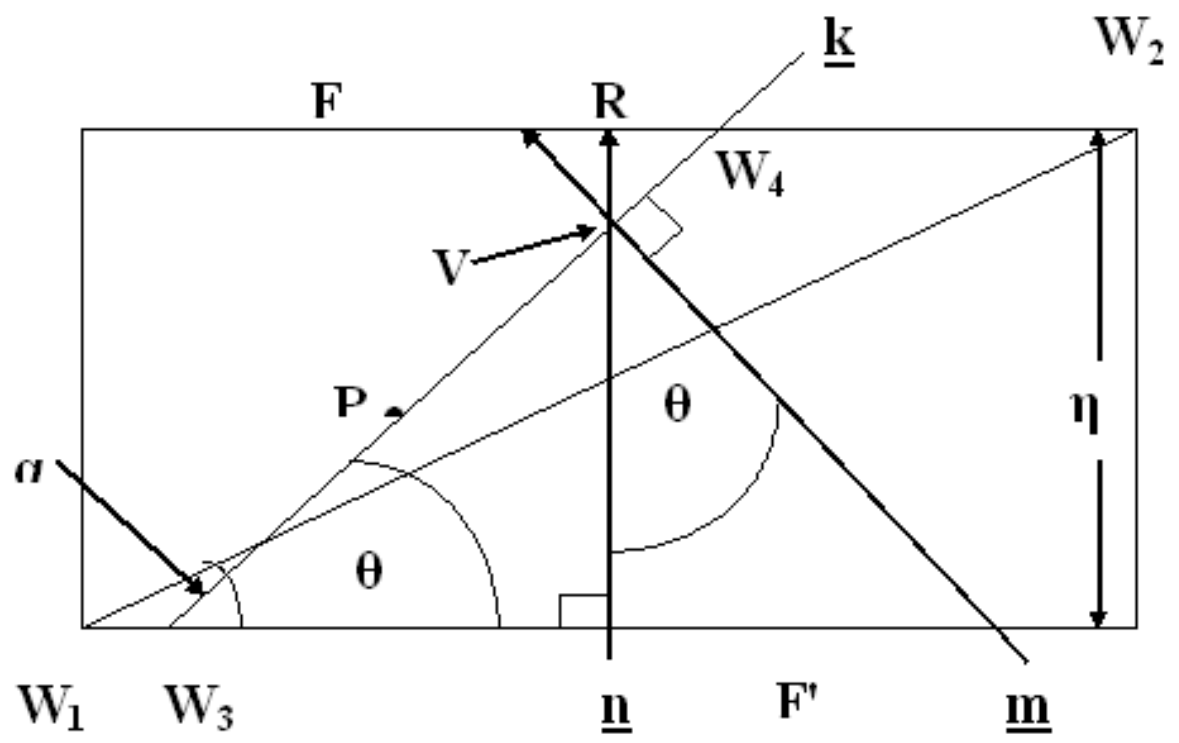

Figure 2:

We can construct in $Y$ a line $\mathbf{m}$, through $V$, that is orthogonal to the line $\mathbf{k}$. By the defintion of completely orthogonal planes, all lines perpendicular to $\mathbf{k}$ and not in $\Pi$ must lie in $\Psi$. Therefore the line $\mathbf{m}$ lies in $Y \cap \Psi$ making an angle $\theta$ with $\mathbf{n}$, the normal to the tangent hyperplane to $C_{1}$ at $R$.

By construction, any vector lying wholly within the $d$-cylinder $G$ has length $\leq W_{1} W_{2}$, so that

$$
W_{3} W_{4}=\eta \operatorname{cosec} \theta \leq W_{1} W_{2}=\eta \operatorname{cosec} \alpha .
$$

By equation (16), the distance of points of $S^{\star}(V)$ from $V$ is at most

$$
r=10 \sqrt{\delta c_{1} M}
$$

so that $S^{\star}(V)$ lies within a distance $\mathrm{r}$ of the line $\mathbf{k}$ in a $j$-dimensional plane $\Pi$. Hence $S^{\star}(V)$ must be contained in a $j$-cylinder, $G^{\prime}$, with axis $k$, whose upper and lower faces are $(j-1)$-spheres of radius $r$. The $j$-dimensional volume of $G^{\prime}$ is therefore

$$
\alpha_{j-1} r^{j-1} W_{3} W_{4}=\alpha_{j-1} r^{j-1} \eta \operatorname{cosec} \theta .
$$

Suppose that the $j$-dimensional boundary component $S^{\star}\left(V_{i}\right)$ contains $l$ points of $S$, where

$$
L+1 \leq l \leq 2 L
$$


for some $L$ equal to a power of two. By Lemma 2.2 in dimension $j$, the convex hull of $S^{\star}(V)$ has $j$-dimensional volume

$$
\operatorname{Vol}\left(S^{\star}(V)\right) \geq \frac{(l-j)}{j !} D(\Lambda) \geq \frac{(L-j+1)}{j !} D(\Lambda) \geq \frac{L}{(j+1) !} D(\Lambda),
$$

where $\left|S^{\star}(V)\right|$ lies in the range of $(68)$.

Comparing (67) and (69), we see that

$$
\sin \theta \leq \frac{(j+1) ! \eta \alpha_{j-1} r^{j-1}}{D(\Lambda) L}
$$

and for acute angles we can write

$$
\theta \leq \frac{\pi}{2} \sin \theta \leq \frac{\pi(j+1) ! \eta \alpha_{j-1} r^{j-1}}{2 D(\Lambda) L}
$$

As stated before, a $j$-girdle is a set of $j$-dimensional boundary components whose $j$-planes $\Pi$ are all completely parallel. We want to count the number of components in the girdle for which (68) holds for each $L$ equal to a power of two. Each boundary component $S^{\star}\left(V_{i}\right)$ gives rise to a set $A_{i}$ along the surface of the sphere $B$, radius $c_{1} M$, introduced in the proof of Lemma 4.1. The set $A_{i}$ has a centre, the point $W_{i}$ where the outward normal is parallel to the line $V R$ normal to $C_{1}$. Corresponding to the unique pair of completely orthogonal $j$ and $(d-j)$-planes $\Pi$ and $\Psi$ through $V$, there are diametric planes of the sphere $B, \Pi^{\prime}$ parallel to $\Pi$, $\Psi^{\prime}$ parallel to $\Psi$, that form a unique completely orthogonal pair of planes through the centre of $B$. The distance of $W_{i}$ from $\Psi^{\prime}$, measured along the surface of $B$, is $\theta c_{1} M$. The distance of each point of $A_{i}$ from $W_{i}$ is

$$
\leq \sqrt{\frac{c_{0} \delta M}{4}}
$$

so that the distance of each point of $A_{i}$ from the $(d-j)$-plane $\Psi^{\prime}$ is

$$
\leq \theta c_{1} M+\sqrt{\frac{c_{0} \delta M}{4}} \leq 2 \max \left(\theta c_{1} M, \theta_{0} c_{1} M\right)
$$

where

$$
\theta_{0}=\frac{1}{c_{1}} \sqrt{\frac{c_{0} \delta}{4 M}}
$$

There are two cases according to which term gives the maximum in (72). In both cases we consider the maximum $(d-1)$-dimensional surface region available on the surface of the $d$-sphere $B$ and relate this to the minimum surface requirement for each set $A_{i}$ on the surface of $B$. We note that if more than one $j$-dimensional boundary component in a $j$-girdle of the convex hull $H$ lies on the 
same $j$-plane, then the vertices $V_{i}$, which label the boundary components $S^{\star}\left(V_{i}\right)$ must be different, so they are counted separately in this argument.

First we consider $L$ so small that

$$
\frac{\pi(j+1) ! \eta \alpha_{j-1} r^{j-1}}{2 D(\Lambda) L} \geq \frac{\pi}{2} \sin \theta \geq \theta \geq \theta_{0}=\frac{1}{c_{1}} \sqrt{\frac{c_{0} \delta}{4 M}} .
$$

Then

$$
\frac{\pi(j+1) ! \eta \alpha_{j-1} r^{j-1} c_{1} M}{D(\Lambda) L} \geq 2 \max \left(\theta c_{1} M, \theta_{o} c_{1} M\right) .
$$

The intersection of $\Psi^{\prime}$ with $B$ is a $(d-j)$-dimensional sphere, $B_{1}$, with diameter $2 c_{1} M$. The $(d-j-1)$-dimensional surface of $B_{1}$ is contained within the $(d-1)$ dimensional surface of $B$, and by (6) this is given by

$$
(d-j) \alpha_{d-j}\left(c_{1} M\right)^{d-j-1} .
$$

The set $A_{i}$ has distance at most $2 \theta c_{1} M$ from the $(d-j)$-plane $\Psi^{\prime}$ on the surface of $B$ in $j$ further perpendicular directions, and so has cross-section at most $4 \theta c_{1} M$ in these $j$ dimensions. Hence the search region on the surface of $B$ has $(d-1)$ dimensional volume at most

$$
\begin{gathered}
(d-j) \alpha_{d-j}\left(c_{1} M\right)^{d-j-1}\left(4 \theta c_{1} M\right)^{j} \leq\left(2 \pi c_{1} M\right)^{d-j-1}\left(4 \theta c_{1} M\right)^{j} \\
\leq\left(2 \pi c_{1} M\right)^{d-j-1}\left(\frac{2 \pi(j+1) ! \eta \alpha_{j-1} r^{j-1} c_{1} M}{D(\Lambda) L}\right)^{j}
\end{gathered}
$$

where we have used (8). By (30), the number of such sets $A$ is at most

$$
\begin{gathered}
\frac{1}{\alpha_{d-1}}\left(\sqrt{\frac{4}{c_{0} \delta M}}\right)^{d-1}\left(2 \pi c_{1} M\right)^{d-j-1}\left(\frac{2 \pi(j+1) ! \eta \alpha_{j-1} r^{j-1} c_{1} M}{D(\Lambda) L}\right)^{j}= \\
\left(\frac{2^{2(d-1)+j^{2}} 5^{j(j-1)} 13^{j} \alpha_{j-1}^{j} \pi^{d-1}((j+1) !)^{j} c_{1}^{\frac{2 d+j^{2}+j-2}{2}}}{\alpha_{d-1} c_{0}^{\frac{d+2 j-1}{2}}(D(\Lambda) L)^{j}}\right) \delta^{\frac{j^{2}+j-d+1}{2}} M^{\frac{d+j^{2}-j-1}{2}} .
\end{gathered}
$$

The corresponding boundary components $S^{\star}(V)$ have at most $2 L$ points. We then sum over $L=2,4,8, \ldots$ to get a contribution

$$
\leq\left(\frac{2^{2 d+j^{2} 5^{j(j-1)} 13^{j} \alpha_{j-1}^{j} \pi^{d-1}((j+1) !)^{j} c_{1}^{\frac{2 d+j^{2}+j-2}{2}}}}{\alpha_{d-1} c_{0}^{\frac{d+2 j-1}{2}}(D(\Lambda))^{j}}\right) \delta^{\frac{j^{2}+j-d+1}{2}} M^{\frac{d+j^{2}-j-1}{2}}
$$

of points to $S$ from all the boundary components in the girdle in the cases (73).

For ranges of $L$ for which (73) is false we have

$$
\sin \theta \leq \frac{(j+1) ! \eta \alpha_{j-1} r^{j-1}}{D(\Lambda) L}<\frac{2 \theta_{0}}{\pi}=\frac{1}{\pi c_{1}} \sqrt{\frac{c_{0} \delta}{M}},
$$




$$
\begin{gathered}
\theta \leq \frac{\pi}{2} \sin \theta<\theta_{0}=\frac{1}{2 c_{1}} \sqrt{\frac{c_{0} \delta}{M}}, \\
2 \max \left(\theta c_{1} M, \theta_{o} c_{1} M\right)<2 \theta_{0} c_{1} M=\sqrt{c_{0} \delta M} .
\end{gathered}
$$

The sets $A_{i}$ corresponding to the extended components with all $L$ for which (73) is false are disjoint, and they lie within a region of $(d-1)$-volume at most

$$
\begin{gathered}
\left(2 \pi c_{1} M\right)^{d-j-1}\left(4 \theta_{0} c_{1} M\right)^{j} \leq\left(2 \pi c_{1} M\right)^{d-j-1}\left(2 \sqrt{c_{0} \delta M}\right)^{j}, \\
=2^{d-1}\left(\pi c_{1}\right)^{d-j-1}\left(c_{0} \delta\right)^{\frac{j}{2}} M^{\frac{2 d-j-2}{2}}
\end{gathered}
$$

using the same reasoning as that of the previous case.

By (30), the number of such sets $A_{i}$ is at most

$$
\begin{gathered}
\frac{1}{\alpha_{d-1}}\left(\sqrt{\frac{4}{c_{0} \delta M}}\right)^{d-1} 2^{d-1}\left(\pi c_{1}\right)^{d-j-1} c_{0}^{\frac{j}{2}} \delta^{\frac{j}{2}} M^{\frac{2 d-j-2}{2}}, \\
=\left(\frac{2^{2 d-2}\left(\pi c_{1}\right)^{d-j-1} c_{0}^{\frac{j+1-d}{2}}}{\alpha_{d-1}}\right) \delta^{\frac{j+1-d}{2}} M^{\frac{d-j-1}{2}} .
\end{gathered}
$$

However small $\theta$ is, the integer points of $S^{\star}(V)$ lie in a $j$-dimensional cube of $j$-volume

$$
\left(20 \sqrt{\delta c_{1} M}\right)^{j}
$$

so if there are $l \geq(j+1)$ integer points in $S^{\star}(V)$, by the minor arc case $d=j$ in Lemma 2.2

$$
\frac{l}{(j+1) !} D(\Lambda) \leq \frac{l-j+1}{j !} D(\Lambda) \leq\left(20 \sqrt{\delta c_{1} M}\right)^{j},
$$

so that,

$$
l \leq \frac{(j+1) !}{D(\Lambda)}\left(20 \sqrt{\delta c_{1} M}\right)^{j}
$$

and the boundary components $S^{\star}(V)$ in the girdle for which (73) is false contribute

$$
\begin{gathered}
\leq \frac{(j+1) !}{D(\Lambda)}\left(20 \sqrt{\delta c_{1} M}\right)^{j} \cdot\left(\frac{2^{2 d-2}\left(\pi c_{1}\right)^{d-j-1} c_{0}^{\frac{j+1-d}{2}}}{\alpha_{d-1}}\right) \delta^{\frac{j+1-d}{2}} M^{\frac{d-j-1}{2}} \\
\quad=\left(\frac{(j+1) ! 2^{2 d+2 j-2} 5^{j} \pi^{d-j-1} c_{0}^{\frac{j+1-d}{2}} c_{1}^{\frac{2 d-j-2}{2}}}{\alpha_{d-1} D(\Lambda)}\right) \delta^{\frac{2 j+1-d}{2}} M^{\frac{d-1}{2}}
\end{gathered}
$$

integer points to $S(H)$. 
We use Lemma 5.2 with $j=k$ to estimate the contribution of all boundary components with $L$ small in all $j$-girdles given by (75) as

$$
\begin{gathered}
\left(\frac{2^{3 j d+2 d+2 j^{2}} 5^{j(d-1)} 13^{j} \alpha_{j-1}^{j} \pi^{d-1}((j+1) !)^{j} c_{1}^{\frac{2 d+j d+j-2}{2}}}{\alpha_{d-1} \alpha_{j}^{j} c_{0}^{\frac{d+2 j-1}{2}}}\right) \\
\times \delta^{\frac{(d+1)(j-1)}{2}+1} M^{\frac{(d-1)(j+1)}{2}}
\end{gathered}
$$

integer points, and the contribution of all boundary components with $L$ large from all $j$-girdles given by (76) as

$$
\left(\frac{(j+1) ! 2^{3 j d+3 j+2 d-2} 5^{j d} \pi^{d-j-1} c_{0}^{\frac{j+1-d}{2}} \frac{j d+2 d-2 j-2}{2}}{c_{1}}\right) \delta^{\frac{(d+1)(j-1)}{2}+1} M^{\frac{(d-1)(j+1)}{2}} .
$$

After some calculation we find that

$$
\begin{gathered}
\frac{\frac{2 d+j d+j-2}{2}}{\frac{c_{1} \frac{d+2 j-1}{2}}{c_{0}}} \geq c_{0}^{\frac{j+1-d}{2}} c_{1}^{\frac{j d+2 d-2 j-2}{2}}, \\
\frac{\alpha_{j-1}}{\alpha_{j}} \leq j,
\end{gathered}
$$

and

$$
(j(j+1) !)^{j} \geq \frac{(j+1) !}{\alpha_{j}},
$$

for all $j \geq 0, d \geq 1$, where we have used (4) (6) and (7) to obtain the above inequalities. Hence we can write the sum of these two terms from (77) and (78) as

$$
\leq \lambda_{j}\left(\frac{c_{1}^{2}}{c_{0}^{2}} \delta^{d+1}\left(c_{1} M\right)^{d-1}\right)^{\frac{j-1}{2}}\left(\left(\frac{c_{1}}{c_{0}}\right)^{\frac{d+1}{2}} \delta\left(c_{1} M\right)^{d-1}\right)
$$

where we have written

$$
\lambda_{j}=\left(\frac{2^{3 j d+2 d+2 j^{2}+2 j}\left(5^{j} \pi\right)^{d-1}(9 j(j+1) !)^{j}}{\alpha_{d-1}}\right) .
$$

We now consider the total number of integer points contributed by the $j$-girdles in all boundary components with $\delta \leq \delta_{0}$, defined in (17). Hence

$$
\delta^{d+1}<\delta_{0}^{d+1}=\left(\frac{c_{0}}{2^{2 d} 5^{d-1} 13 d ! c_{1}}\right)^{2}\left(c_{1} M\right)^{-(d-1)}
$$


and

$$
\left(\frac{c_{1}^{2}}{c_{0}^{2}} \delta^{d+1}\left(c_{1} M\right)^{d-1}\right)^{\frac{j-1}{2}} \leq\left(\frac{1}{2^{2 d} 5^{d-1} 13 d !}\right)^{j-1}=\mu_{j}
$$

say, where $\mu_{j}$ is a constant depending only on $d$ and $j$.

In this notation, the upper bound in (79) for the components with $\delta \leq \delta_{0}$ is

$$
\lambda_{j} \mu_{j}\left(\left(\frac{c_{1}}{c_{0}}\right)^{\frac{d+1}{2}} \delta\left(c_{1} M\right)^{d-1}\right) .
$$

Using the inequalities

$$
\begin{gathered}
\frac{9^{j}}{13^{j-1}} \leq 9, \quad j \geq 1, \\
\frac{j^{j}(j+1) !^{j}}{d !^{j-1}} \leq d !, \quad j \leq d-2,
\end{gathered}
$$

we can write

$$
\lambda_{j} \mu_{j} \leq \frac{2^{8 d+3 j d+2 j} d !}{\alpha_{d-1}} .
$$

Now

$$
\sum_{j=2}^{d-2} 2^{2 j}=\frac{\left(2^{d}-8\right)\left(2^{d}+8\right)}{12} \leq 2^{2 d-3}
$$

and

$$
\sum_{j=2}^{d-2} 2^{3 j d}=\frac{2^{3 d^{2}}-2^{6 d}}{2^{6 d}-2^{3 d}} \leq 2^{3 d^{2}-5 d} .
$$

Hence we estimate the contribution of integer points from all $j$-dimensional girdles, with $2 \leq j \leq(d-2)$, and $\delta \leq \delta_{0}$ as

$$
N_{g} \leq\left(\frac{2^{3 d^{2}+5 d-3} d !}{\alpha_{d-1}}\right)\left(\left(\frac{c_{1}}{c_{0}}\right)^{\frac{d+1}{2}} \delta_{0}\left(c_{1} M\right)^{d-1}\right) .
$$

Next, for $\delta \leq \delta_{0}$, we consider the integer points contributed by the boundary components of dimension $0,1, d-1$ and $d$, along with the points lying strictly inside the convex hull $H$. These individual upper bounds correspond to (25),(27), (34), (60) and (23) respectively, and adding them we have

$$
\leq 2^{\frac{d^{2}+10 d+18}{2}} d !\left(\frac{c_{1}}{c_{0}}\right)^{\frac{d-1}{2}}\left(\left(c_{1} M\right)^{\frac{d(d-1)}{d+1}}+\frac{2^{4}}{\alpha_{d-1}}\left(\frac{c_{1}}{c_{0}}\right)^{\frac{d-1}{2}} \delta_{0}\left(c_{1} M\right)^{d-1}\right)
$$

integer points. Combining (81) with (82) then gives the total number of integer points lying on within a distance $\delta_{0}$ from the convex hull $H$ as

$$
\leq 2^{\frac{d^{2}+10 d+18}{2}} d !\left(\frac{c_{1}}{c_{0}}\right)^{\frac{d-1}{2}}\left(\left(c_{1} M\right)^{\frac{d(d-1)}{d+1}}+\frac{2^{\frac{5 d^{2}-22}{2}}}{\alpha_{d-1}}\left(\frac{c_{1}}{c_{0}}\right)^{\frac{d-1}{2}} \delta_{0}\left(c_{1} M\right)^{d-1}\right)
$$


This result is valid for a shell of thickness $\delta=\delta_{0}$ and consists of terms independent of $\delta$ (degree zero), and those with a factor of $\delta$ (degree one).

We cover the shell $E$ of all extended vertex components, bounded internally by $C_{0}$ and externally by $C_{1}$, by $R$ thinner concentric shells $E_{1}, \ldots, E_{R}$ of thickness $\delta_{0}$. The distance between $C_{1}$ and $C_{0}$ along any inward normal vector to these two surfaces is $2 \delta$. Hence we choose $R$ to be the smallest such integer with

$$
R \delta_{0} \geq 2 \delta, \quad(R-1) \delta_{0}<2 \delta
$$

so that

$$
R<\frac{2 \delta}{\delta_{0}}+1
$$

The shell $E_{r}$ consists of the points on some inward normal whose distance $l$ from the hypersurface $C_{1}$ lies in the range

$$
(r-1) \delta_{0} \leq l \leq r \delta_{0}
$$

Replacing $\delta$ with $r \delta_{0}$ in Lemma 2.1 implies that each shell $E_{r}$ will satisfy the Curvature Condition, so that any two-dimensional plane sectional curve of $E_{r}$ will lie in the range

$$
c_{0} M \leq \rho \leq c_{1} M
$$

Therefore, equation (83) gives a uniform upper bound for the number of integer points contributed by any shell $E_{r}$. Now let

$$
\eta=\delta_{0}\left(c_{1} M\right)^{\frac{d-1}{d+1}}=\left(\frac{c_{0}}{2^{2 d} 5^{d-1} 13 d ! c_{1}}\right)^{\frac{2}{d+1}} \leq \frac{1}{2^{6}} .
$$

Then

$$
\frac{1}{\eta}=\left(\frac{2^{2 d} 5^{d-1} 13 d ! c_{1}}{c_{0}}\right)^{\frac{2}{d+1}} \leq 2^{d+8} \frac{c_{1}}{c_{0}}
$$

and

$$
\delta_{0}=\frac{\eta}{\left(c_{1} M\right)^{\frac{d-1}{d+1}}} \leq \frac{1}{2^{6}\left(c_{1} M\right)^{\frac{d-1}{d+1}}} .
$$

We are now ready to prove Theorems 1.1 and 1.2 .

Proof of Theorem 1.1. We multiply the upper bound (83) by the maximum number of shells allowed by (84). For the degree zero terms this yields

$$
\begin{gathered}
\leq\left(\frac{2 \delta}{\delta_{0}}+1\right) 2^{\frac{d^{2}+10 d+18}{2}} d !\left(\frac{c_{1}}{c_{0}}\right)^{\frac{d-1}{2}}\left(c_{1} M\right)^{\frac{d(d-1)}{d+1}} \\
\leq \frac{2^{\frac{d^{2}+10 d+18}{2}}}{\eta}\left(\frac{c_{1}}{c_{0}}\right)^{\frac{d-1}{2}} \delta\left(c_{1} M\right)^{d-1}+2^{\frac{d^{2}+10 d+18}{2}} d !\left(\frac{c_{1}}{c_{0}}\right)^{\frac{d-1}{2}}\left(c_{1} M\right)^{\frac{d(d-1)}{d+1}}
\end{gathered}
$$




$$
\leq 2^{\frac{d^{2}+12 d+34}{2}} d !\left(\frac{c_{1}}{c_{0}}\right)^{\frac{d+1}{2}} \delta\left(c_{1} M\right)^{d-1}+2^{\frac{d^{2}+10 d+18}{2}} d !\left(\frac{c_{1}}{c_{0}}\right)^{\frac{d-1}{2}}\left(c_{1} M\right)^{\frac{d(d-1)}{d+1}}
$$

For the degree one terms we have

$$
\begin{gathered}
\leq\left(\frac{2 \delta}{\delta_{0}}+1\right) \frac{2^{3 d^{2}+5 d-2} d !}{\alpha_{d-1}}\left(\frac{c_{1}}{c_{0}}\right)^{d-1} \delta_{0}\left(c_{1} M\right)^{d-1} \\
\leq \frac{2^{3 d^{2}+5 d-1} d !}{\alpha_{d-1}}\left(\frac{c_{1}}{c_{0}}\right)^{d-1} \delta\left(c_{1} M\right)^{d-1}+\frac{2^{3 d^{2}+5 d-2} d !}{\alpha_{d-1}}\left(\frac{c_{1}}{c_{0}}\right)^{d-1} \eta\left(c_{1} M\right)^{\frac{d(d-1)}{d+1}} \\
\leq \frac{2^{3 d^{2}+5 d-1} d !}{\alpha_{d-1}}\left(\frac{c_{1}}{c_{0}}\right)^{d-1} \delta\left(c_{1} M\right)^{d-1}+\frac{2^{3 d^{2}+5 d-8} d !}{\alpha_{d-1}}\left(\frac{c_{1}}{c_{0}}\right)^{d-1}\left(c_{1} M\right)^{\frac{d(d-1)}{d+1}}
\end{gathered}
$$

Finally we combine the terms from (88) and (89) to estimate the total number of integer points by

$$
\leq \frac{2^{3 d^{2}+5 d-7} d !}{\alpha_{d-1}}\left(\frac{c_{1}}{c_{0}}\right)^{d-1}\left(\left(c_{1} M\right)^{\frac{d(d-1)}{d+1}}+2^{9} \delta\left(c_{1} M\right)^{d-1}\right),
$$

as required.

Proof of Theorem 1.2. In the proof of Theorem 1.1, we consider an enlarged component $S^{\prime}(V)$, where all the calculations for distances between points on the outer surface $C_{1}$ take place within the reach $R(V)$ of $S^{\prime}(V)$, with respect to $V$.

By Lemma 3.7, the Local Curvature Condition holds at all points in $R(V)$, so the calculations which establish Theorem 1.1 are valid under the weaker hypothesis of the Local Curvature Condition.

\section{References}

[1] G. E. Andrews, An asymptotic expression for the number of solutions of a general class of diophantine equation, Trans. Amer. Math. Soc. 99, 1961, 272-277.

[2] G. E. Andrews, A lower bound for the volume of strictly convex bodies with many boundary lattice points, Trans. Amer. Math. Soc. 106, 1963, 270-279.

[3] M. Beck, J. A. De Loera, M. Develin, J. Pfeifle, and R. P. Stanley, Coefficients and Roots of Ehrhart Polynomials, Contemporary Mathematics 374, Amer.Math.Soc. 2005, 15-35.

[4] M. Beck, and S. Robins, Computing the Continuous Discretely, Springer, Heidelberg, 2006.

[5] M. Branton and P. Sargos, Points entiers au voisinage d'une courbe plane à trés faible courbure, Bull. Sci. Math. 118, 15-28. 
[6] M. Filaseta and O. Trifanov, The distribution of fractional parts with applications to gap results in number theory, Proc. London. Math. Soc. (3) 73, 1996, 241-278.

[7] P. M. Gruber, and C. G. Lekkerkerker, Geometry of Numbers, North-Holland Mathematical Library, 1987.

[8] E. Hlawka, Integrale auf konvexen Körpern, Monatshefte Math. 54, 1950, 1-36, 81-99.

[9] M. N. Huxley, Area, Lattice Points and Exponential Sums, Oxford University Press, 1996.

[10] M. N. Huxley, The integer points close to a curve III, in: Number Theory in Progress, K. Györy et al (ed.), de Gruyter, Berlin, 1999, Vol II, 911-940.

[11] M. N. Huxley and P. Sargos, Points entiers au voisinage d'une courbe plane de classe $C^{n}$, Functiones et Approximatio XXXV (2006), 91-115.

[12] V. Jarník, Über die Gitterpunkte auf konvexen Kurven, Math. Zeitschrift 24, $1925,500-518$.

[13] E. Krätzel, Analytische Funktionen in der Zahlentheorie, Teubner, 2000, 200243.

[14] M. C. Lettington, PhD Thesis, Cardiff University, 2008.

[15] M. C. Lettington, Integer points close to convex surfaces, accepted for publication.

[16] P. McMullen and G. C. Shephard, Convex Polytopes and the Upper Bound Conjecture London. Math. Soc. Lecture Note Series 3, Cambridge University Press, 1971.

[17] P. McMullen, The maximum number of faces of a convex polytope, Mathematika 17, 1970, 179-184.

[18] W. Müller, Lattice points in large convex bodies, Montatshefte Math. 128, 1999, 315-330.

[19] D. M. Y. Sommerville, An Introduction to the Geometry of N Dimensions, Methuen, London, 1929.

School of Mathematics

Cardiff University

P.O. Box 926

Cardiff CF24 4YH

UK

E-Mail: LettingtonMC@cf.ac.uk;matt.lettington@sky.com 